\title{
Measurement of lubrication condition and oil film thickness with using ultrasonic technique
}

\author{
Akitoshi Takeuchi ${ }^{1}$, Seiichi Terada ${ }^{2}$ and So Toda ${ }^{2}$ \\ 1. Department of Intelligent Mechanical Systems Engineering, Kochi University of Technology, \\ Tosayamada-chou, Kochi, Japan \\ 2. Automax Co., Ltd., Itabashi-ku, Tokyo, Japan
}

\begin{abstract}
An application of ultrasonic technique is attempted for the purpose of measuring film thickness between moving surfaces. The measurement of film thickness is possible only by setting the ultrasonic probe to one of the friction surface, and the drilling for installing the probe like a measurement using a displacement sensor is not conducted. The ultrasonic wave emitted to the hydrodynamic lubrication interface is reflected multiple in oil film, and the echo height of reflected wave from the boundary is dependent on film thickness. For example, the thickness of oil film between cylinder and piston ring can be easily measured only by setting the small ultrasonic probe on the back of piston ring. On the other hand, the echo height under the mixed lubrication region is mainly dependent on the solid contact area. Therefore, the load share rate at the solid contact parts can be estimated from observed echo height.
\end{abstract}

Key words: Oil film thickness, Load share rate, Mixed lubrication, Ultrasonic technique, Piston ring,

\section{INTRODUCTION}

For enhancement of automotive fuel consumption, an improvement of a mixed lubrication characteristic of a piston ring is one of the important subjects. A wide variety of surface coatings can be applied to piston ring surface depending upon the lubrication conditions. It is desirable to evaluate the lubrication capabilities of these coatings in terms of both the behavior of the lubricating oil film and of the load sharing ratio for the solid-to-solid contact, with using the identical sensor.
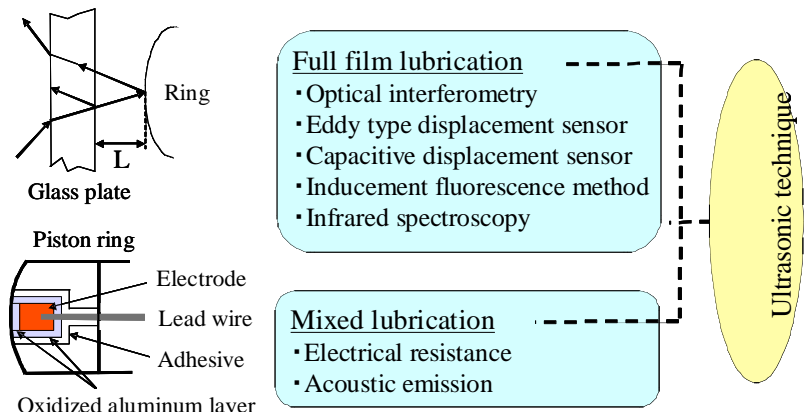

Fig.1 Example of measurement technique

For evaluation of lubricated condition, there are various methods shown in Fig.1. When the film thickness is measured by displacement sensor such as an eddy current or a capacity, sensor is implanted in ring, and it is necessary to reconcile the forefront of sensor with ring surface $[1,2]$. In addition, it is difficult to apply to the piston ring with thickness of about $1.2 \mathrm{~mm}$, wide used for gasoline engine generally. Furthermore, the load sharing rate of a solid contact in a mixed lubrication region can- not be determined by displacement sensor described above.

In this study, the performance of piston rings was evaluated using ultrasonic pulses for the purpose of easily measuring film thickness. Measurement of film thickness becomes possible only by setting a ultrasonic probe at backside of piston ring, and the drilling for installing the probe like a displacement sensor is not conducted $[3,4]$.

In this paper, film thickness and load sharing rate at the solid contact for the piston ring estimated by ultrasonic method under room temperature and atmospheric pressure are described.

\section{PRINCIPLES OF OBSER VATIONS}

As shown in Fig.2, when an ultrasonic pulse is irradiated into an oil film and film thickness is less than the pulse width, multiple reflections will be occurred. Then, the echo height $h$ of the first reflected waves changes in response to the oil film thickness. It will then be pos-
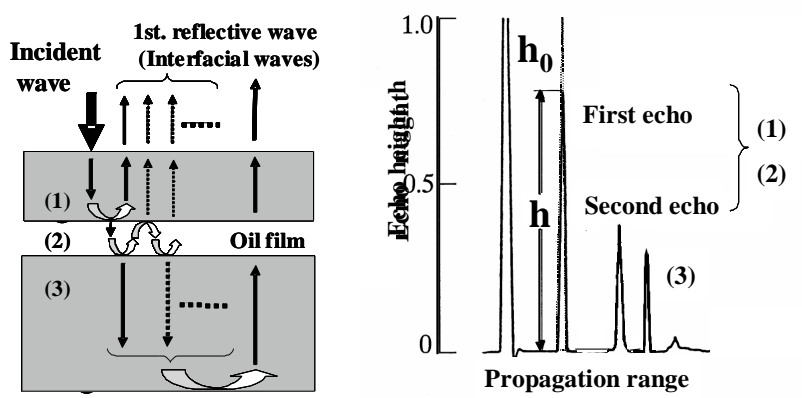

sible to estimate the film thickness from the observed $h$. 


\section{Fig.2 Principle of experimental observation}

For the evaluation of lubrication conditions, echo height ratio, $\mathrm{H}=\mathrm{h} / \mathrm{h}_{0} \times 100 \%$, is defined. In here, $\mathrm{h}$ is the echo height observed in operation, and $h_{0}$ is echo height in dry condition.

On the other hand, in mixed lubrication region, the load is supported by the oil film and solid contact parts as shown in Fig.3. In this lubrication region, observed echo height variation is mainly affected by solid contact points, because the supporting load with solid contact parts increases rapidly even by slightly decrease of a film thickness. In usually, the echo height ratio $\mathrm{H}$ is proportional to the solid contact area $\mathrm{A}$, and it is proportional to

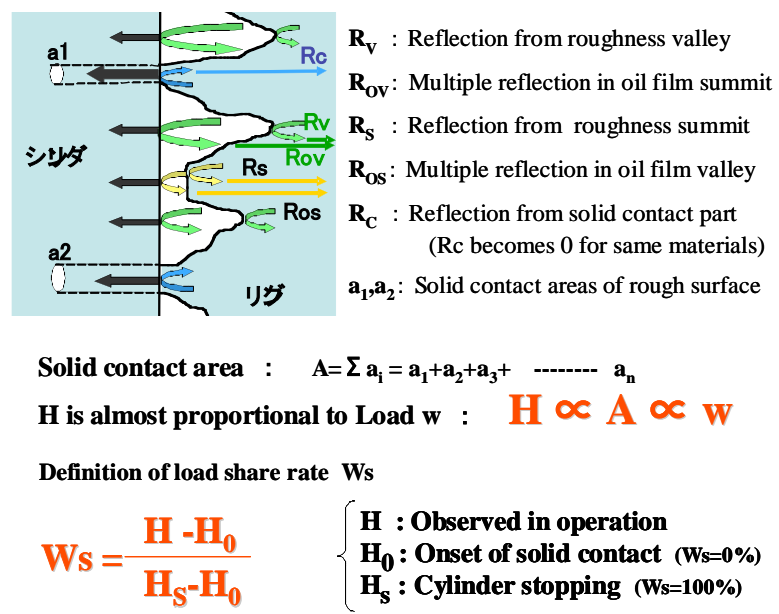

the load W which is supported by solid contact points.

\section{Fig.3 Reflection of sound under mixed lubrication}

Then, the load share rate Ws can be supposed by $\left(\mathrm{H}-\mathrm{H}_{0}\right) /\left(\mathrm{Hs}-\mathrm{H}_{0}\right)$, because they have a linear relationship. In here, $\mathrm{H}$ shows observed echo height ratio in operation, $\mathrm{H}_{0}$ is the onset of solid contact, and the echo height ratio when all loads are supported by solid contact is $\mathrm{H}_{\mathrm{S}}$. Therefore, at $\mathrm{H}=\mathrm{H}_{0}$ point, the load share rate $\mathrm{W}_{\mathrm{S}}$ is $0 \%$, and it becomes to $100 \%$ when $\mathrm{H}=\mathrm{H}_{\mathrm{S}}$.

\section{TEST RING AND CALIBRATION OF FILM THICKNESS}

Principal dimensions of piston ring and cylinder used for the experiment, materials, tensile force and various value of probe are shown in Table 1. The piston ring width used here is $1.2 \mathrm{~mm}$ which is wide used for gasoline engine generally. The crowning of about $4 \mu \mathrm{m}$ has been formed on the piston ring surface. In addition, the crossing roughness, good for lubrication, has been formed on the inside of the cylinder by the honing.

\begin{tabular}{|c|c|c||c|c|}
\hline & Top ring & Cylinder & \multicolumn{2}{c|}{ Composite element } \\
\hline Outer diameter & $86 \mathrm{~mm}$ & $\mathbf{9 6 m m}$ & Frequency & $5 \mathrm{MHz}$ \\
\hline Inner Diameter & $\mathbf{8 0}$ & $\mathbf{8 6}$ & Band width & Wide \\
\hline Thickness & 3 & 5 & Length & $3 \mathrm{~mm}$ \\
\hline Width & 1.2 & ---- & Width & 1 \\
\hline Materials & SWOS-V & S45C & Height & $\mathbf{0 . 5}$ \\
\hline Tension & $6.0 \pm 1 \mathrm{~N}$ & --- & Weight & $\fallingdotseq 1 \mathrm{~m} \mathrm{~N}$ \\
\hline
\end{tabular}

Table 1 Specification of test piece and probe

A small ultrasonic probe is attached to the back side of the piston ring as shown in Fig.4. A composite element is used for probe, and it is cemented on isolation film glued to the inner surface of ring. And backing is applied to attenuate the sound propagated to backside of a composite element. Besides, it is confirmed that the reflected wave from oil film region is not influenced by the

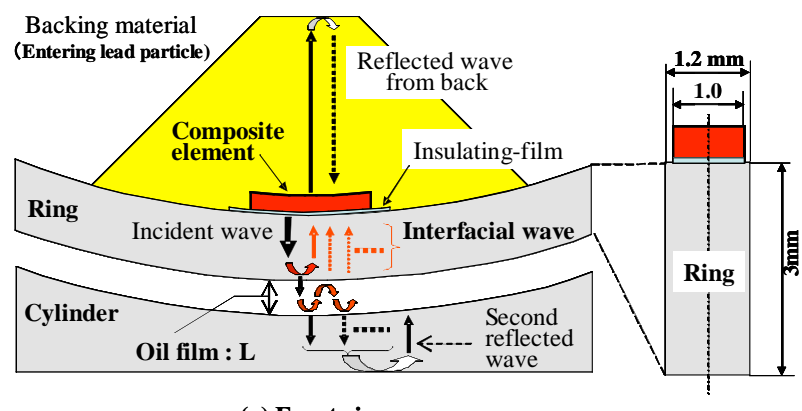

(a) Front view

(b) Side view

edge or the side face of the piston ring.

Fig.4 Probe for $1.2 \mathrm{~mm}$ piston ring

The relationship between the echo height ratio $\mathrm{H}$ (= $\left.\mathrm{h} / \mathrm{h}_{0} \times 100, \%\right)$ and the film thickness $\mathrm{L}$ can be found by using the calibrator shown in Fig.5. The film thickness calibrator of Fig. 5 is the structure that oil bath goes up and down with a micrometer head, and the relations between the film thickness and echo height can be measured. And, the load cell is placed under the oil bath in order to know the relationship between echo height and ring load,

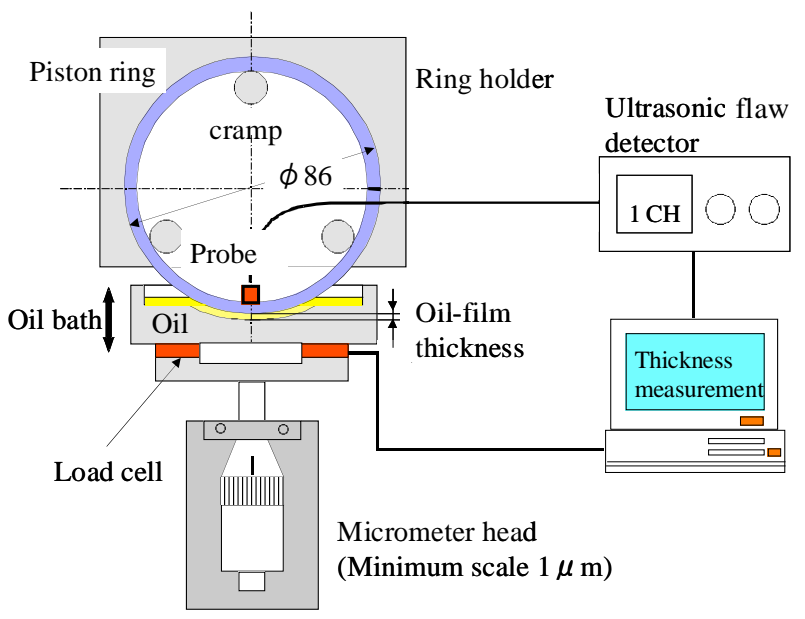

after the ring contacted oil bath.

Fig.5 Film thickness calibrator

Figure 6 is the calibration curve of echo height ratio $\mathrm{H}$ and film thickness L. Because decreasing of $\mathrm{H}$ in region of thin oil film less than $5 \mu \mathrm{m}$ is sudden, the oil film thickness can be estimated with high accuracy from the echo height ratio $H$. This result is convenient for measuring of the film thickness in the top and bottom dead centre which is important for lubrication of piston ring. 
In addition, calibration curve after experiment ( mark) agrees with before experiment ( mark) approximately, and good reproducibility is recognized. After the oil bath contacted with the ring, $\mathrm{H}$ decreases with an increase of ring load approximately linearly. Then an approximation curve to fit each data point of $\mathrm{H}$ is provided in polynomial, and a film thickness and load share rate are

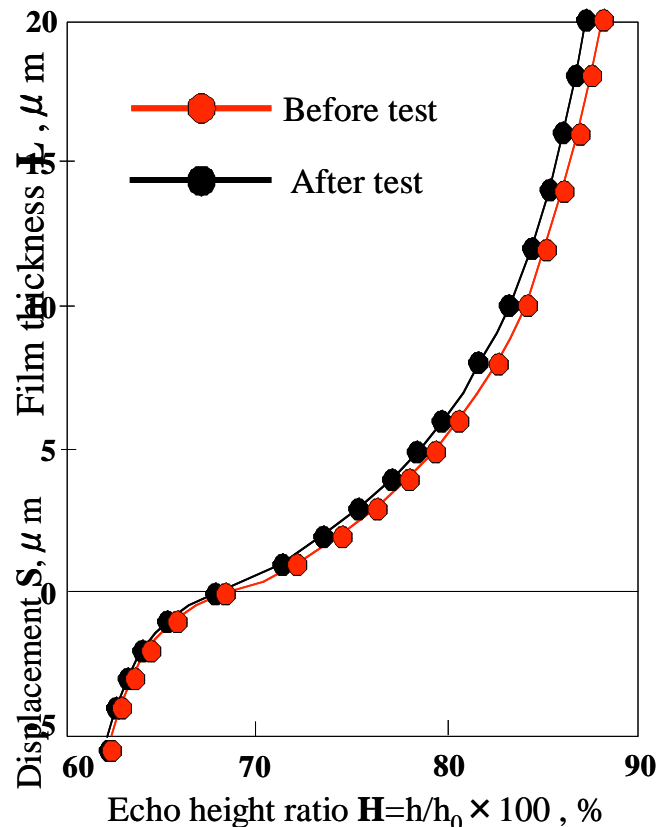

estimated.

\section{Fig.6 Calibration curve}

Fig.7 is a schematic view of the experimental equipment. In this device, cylinder driven with a linear motor moves instead of piston. Therefore, the slap occurring in a normal piston motion does not need to consider, and understanding of a change of a film thickness becomes easy. Stroke length of cylinder is $\pm 50 \mathrm{~mm}$. The experiment is carried out with Low oscillation frequency, $0.25 \sim 1.5 \mathrm{~Hz}$, to clarify lubricated condition near
Fig.7 Schematic view of experiment apparatus

Film thickness is measured only at a top ring, and film thickness variations of top ring by adding a second and oil ring are discussed.

\section{DIFFERENCE OF FILM THICKNESS WITH ADD OF SECOND RING AND OIL RING}

Example of behaviors of oil film thickness on top ring when drive frequency of cylinder is $0.5 \mathrm{~Hz}$, maximum sliding velocity is $0.1574 \mathrm{~m} / \mathrm{s}$, is shown in Fig.8. Oil film behavior is measured with turning a piston ring by 45 degrees in circumferential direction. The behavior in each angle shown in a figure resembles, and enough re-

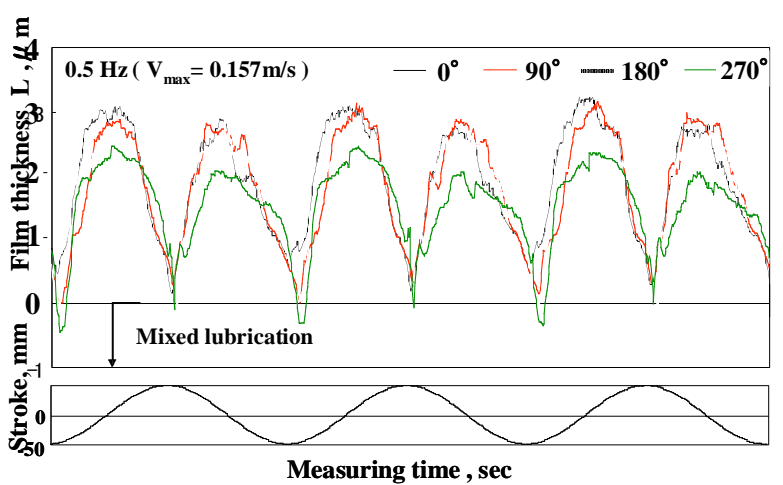

producibility is able to be confirmed.

Fig.8 Behavior of oil film thickness on top ring

Fig.9 shows the distribution of oil film thickness L and load share rate $\mathrm{W}_{\mathrm{S}}$ on reciprocating surface with the angular position $\theta$, and with the range of up and down stroke, $\pm 50 \mathrm{~mm}$. General behavior of film thickness, the film thickness near the center of stroke, $0 \mathrm{~mm}$, is thick whereas the film thickness at top and bottom dead center, $\pm 50 \mathrm{~mm}$, is thin, is observed.

It can be observed distinctly that the oil film thickness decreases by adding a second ring and oil ring to a top

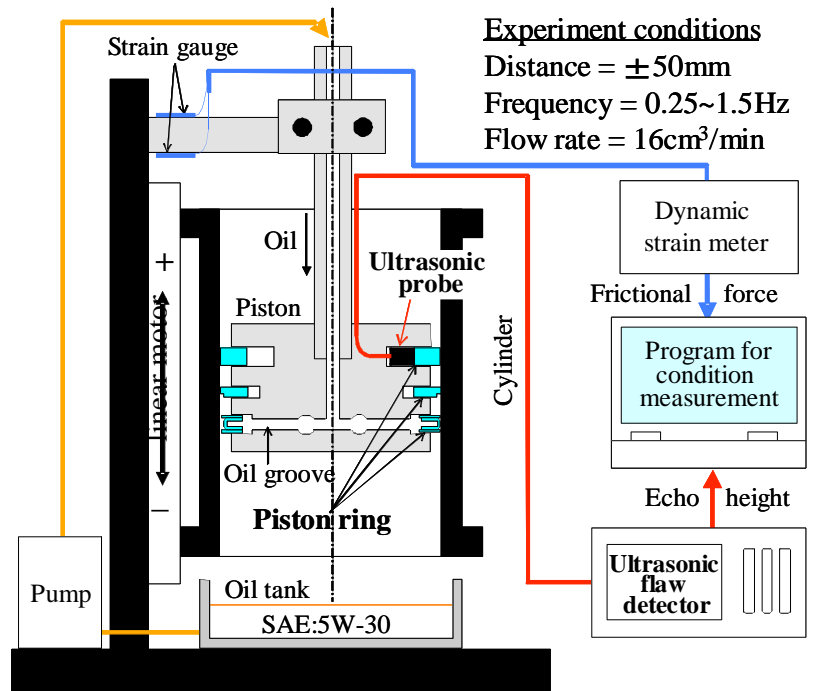

ring, and load share rate with a solid contact part increas- 
es with it to the contrary. However, thickness of oil film is not constant in circumferential direction.

\section{Fig.9 Distribution of $\mathrm{L}$ and Ws}

Then the film thickness in each stroke position is equalized in circumferential direction, and result is shown in Fig.10. The vertical scale of this figure corresponds to lubricated condition, the scale of left side shows oil film thickness and the right side is estimated load share ratio $\mathrm{W}_{\mathrm{S}}$ of top ring under mixed lubrication.

\section{Fig.10 Behavior of equalized $L$ and Ws}

It is clear from this figure that film thickness becomes thin with drop of the drive frequency of cylinder, and lubricated condition shifts to mixed lubrication when the velocity of cylinder is extremely low. Especially, the sliding speed near the top and bottom dead center is considerably low, therefore these locations have wider mixed lubrication regions when all rings are mounted.

Nevertheless $\mathrm{W}_{\mathrm{S}}$ suddenly decreases with incrementation of a sliding velocity, and lubricated condition returns to complete fluid lubrication during a short time.

Therefore, it is easy to understand, the effect of oil film is large, even if the sliding surfaces are operated in the mixed lubrication region.

\section{CONCLUSIONS}

The pulse ultrasonic wave is emitted from the back side of the top ring to the lubricating surfaces, and the film thickness is estimated by reflected echo height from there. As the result, the followings become clear.

1) The relations of echo height and film thickness can be determined by employing the echo height ratio $\mathrm{H}$ standardized with the echo height $h_{0}$ in a dry condition.

2) The difference of the formation condition of oil film against a $1.2 \mathrm{~mm}$ width piston ring can be measured quantitatively.

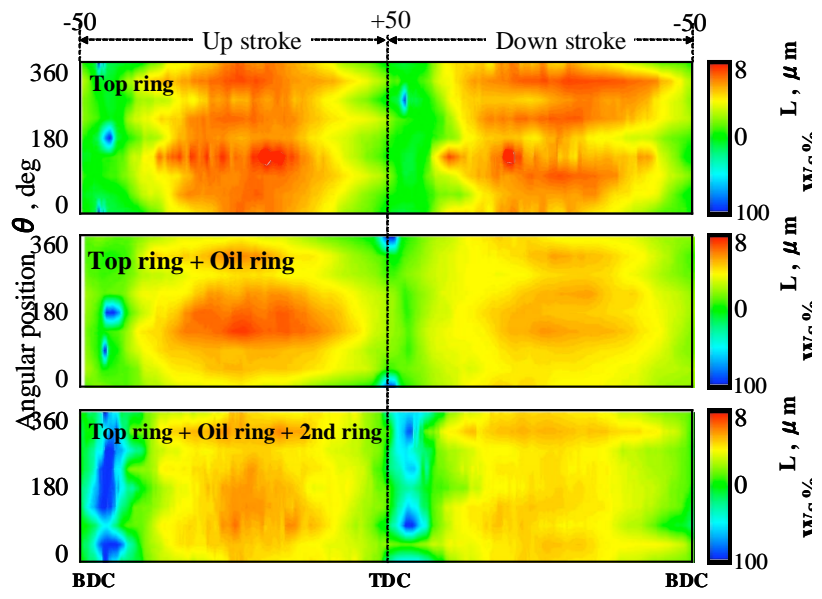

3) The distribution map of film thickness and load share ratio between piston ring and cylinder can be obtained easily.

4) It is necessary to evaluate lubricating characteristic of piston ring with the film thickness which is equalized in each angular position in circumferential direction.

5) There is a potential that can measure the quantitative

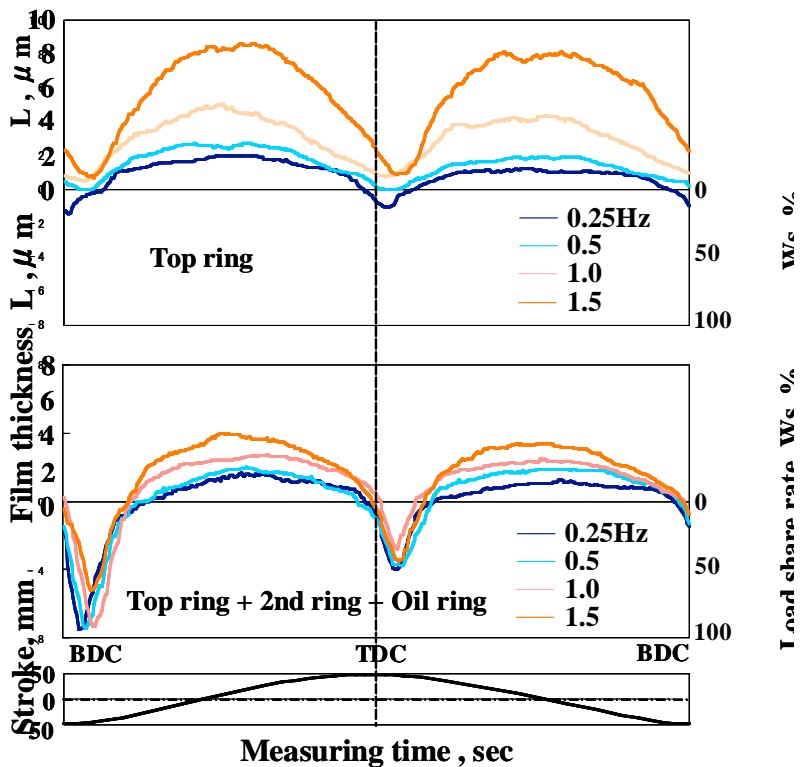

load share ratio in the solid contact region under the mixed lubrication.

\section{REFERENCES}

1. Keiji KYOUGOKU, Tstsuro NAGANUMA, YuichiHAMAMURA, Tsunamitsu NAKAHARA, " Lubrication of Oil-Hydraulic Piston Pump/Motor (Part 2)", Journal of Japan Society of Tribologists, Vol.36,No1, 1989.

2. S.R.Brown, G.M.Hamilton, "The Partially Lubricated Piston Ring”, Journal Mechanical Engineering Science, Vol.19, No2, 1977.

3. A.TAKUHCHI, S.TERADA, S.TODA, T.MIAKE, "Simple Measurement of Film Thickness of Piston Ring by Ultrasonic Method", Proceedings of JAST Tribology Conference Niigata, Nov., 2003.

4. T.MIAKE, A.TAKUHCHI, S.TERADA, S.TODA, "Evaluation of Oil Film Formation Condition in Running-in Process of Piston Ring by Ultrasonic Method", Proceedings of JAST Tribology Conference Tottori, Nov., 2004. 